\title{
Indicadores de prioridade para políticas públicas dirigidas ao desenvolvimento sustentável na Amazônia
}

\author{
Uma construção orientada a sistemas de produção \\ do Nordeste Paraense
}

\author{
Francisco de Assis Costa \\ Professor e pesquisador do Núcleo de Altos \\ Estudos Amazônicos da Universidade \\ Federal do Pará.
}

\section{Resumo}

Este trabalho traz elementos para avaliar a esperança de sustentabilidade de sistemas de produção rural presentes na Amazônia. Faz uma caracterização desses sistemas e seus fundamentos produtivos e reprodutivos na mesorregião Nordeste Paraense, a mais densamente povoada do Estado do Pará, e constrói indicadores que incorporam, além de variáveis econômicas, variáveis que enunciam esperança de maior eficiência ecológica. Pretende contribuir com novas perspectivas para as discussões sobre desenvolvimento e oferecer instrumentos para orientar as instituições que decidem por critérios de sustentabilidade.

\section{Palavras-chave}

Amazônia - Agricultura Desenvolvimento - Sustentabilidade Indicadores.

\begin{abstract}
This article brings elements to estimate the hope of sustainability of present agrarian systems in the Amazonian. It makes a characterization of those systems and their productive and reproductive grounds in the Northeast Region, the most densely populated of the Pará State, and it builds indicators that incorporate, besides economical variables, variables that inform about the ecological efficiency. It intends to contribute with new perspectives for the discussions on socioeconomic development and to offer instruments to guide the institutions that decide for sustainability criteria.
\end{abstract}

\section{Keywords}

Amazon - Agriculture -

Development - Sustainability Indicators - 


\section{Introdução}

Duas coisas têm marcado a discussão ambientalista recente sobre a Amazônia. A primeira é que, curiosamente, a discussão sobre o agrário, nessa ótica, tem-se feito sem maior interação com a discussão sobre sustentabilidade. A segunda é que as noções de sustentabilidade, quando acionadas, estão bem pouco impregnadas de conteúdo social. Em termos formais: os modelos que têm orientado a discussão sobre o uso agropecuário e extrativo dos recursos naturais não incorporam os resultados dos modelos que operacionalizam noções de sustentabilidade, os quais, por seu turno, têm tido dificuldade de internalizar variáveis que explicitem as determinações econômico-sociais na conformação, desenvolvimento e crise dos sistemas ecológicos.

Há, por trás disso, questões de método. Primeiro, as resultantes das formas diferenciadas de delimitação de objeto: para os que vêm estudando a dinâmica agropecuária, a matéria a observar constitui-se de uma polaridade entre desmatamento (ação humana genérica = ação antrópica) e o que foi por ele negado, o objeto do desmatamento (floresta originária $=$ natureza para si); para os que estudam a sustentabilidade, a matéria a considerar forma-se na interação entre o resultado da ação humana e o seu devir: trata-se de saber se, ou até onde, a ecologia montada artificialmente pela ação humana permanecerá. Segundo, associados ao objeto, definem-se planos diferentes de observação: o plano da observação da ação humana genérica contra uma natureza para si é necessariamente macro (repercussão nos "serviços ecológicos", efeitos na ecologia global, etc.) enquanto a observação da sustentabilidade se dá em nível micro, na análise dos artefatos, das plantas industriais, dos sistemas agrícolas, etc.

Em todos os casos, as análises têm excluído o momento social dos processos por se fazerem a partir de contabilidades estanques (fluxos de nutrientes, fluxo energético, etc.), descontextualizadas historicamente, a cujos resultados se atribuem significados absolutos.

Não têm sido triviais as limitações impostas por esse estado de coisas à compreensão do quadro atual e das possibilidades futuras das populações amazônicas. No que se refere à questão recorrente do desmatamento, por exemplo, as dissociações enunciadas têm impedido um melhor tratamento de um grupo de questões essenciais: afinal, em que medida os potenciais efeitos negativos do desmatamento são contra-arrestados pelos sistemas ecológicos resultantes? E, complementarmente, quais os sistemas artificiais que potencialmente refazem equilíbrios perdidos com o desmatamento?

As questões são pertinentes porque, se podemos resolvê-las, alcançamos um outro nível na avaliação do problema: sua extensão passa a definirse pela extensão do desmatamento menos a extensão das áreas com sistemas antrópicos inócuos para o equilíbrio global - isto é, a extensão da transformação da natureza originária menos a extensão dos usos sociais sustentáveis da base natural. 
Ademais, têm-se tido limites graves em encaminhar duas outras questões associadas: quais sistemas sociais são capazes de gerar tais possibilidades sustentáveis de uso dos recursos naturais? E, isso posto, quais agentes (no sentido econômico e sociológico do termo - conf. Giddens, 1989) são capazes de geri-los? Mais uma vez estamos diante de questões relevantes, porquanto um sistema artificial sustentável não se validará socialmente só pela sua correção e acerto, por mais atraente que ele se coloque à razão tecnocrática (Costa, 1998). As possibilidades de um desenvolvimento equilibrado, embora aumentem com o repertório técnico que possa vir a ser posto à disposição da sociedade pelos que atuam no campo de Ciência e Tecnologia (CGT), dependem estritamente da existência de agentes capazes de gerir uma economia cujos fundamentos da sustentabilidade ecológica sejam fundamentos, também, de economicidade e equilíbrio social.

Os agentes atuam no interior de estruturas que mantêm entre si relações sistemáticas, dominadas por antagonismos de fundo produzidos pela disputa por fatores (primários e superiores - conf. Porter, 1989), mercados e disponibilidades intangíveis e pontuadas por arranjos para uma disposição complementar de governança (conf. Williamson, 1985). As expressões regionalizadas, sempre datadas, dessas relações, as quais fazem a sociedade rural, são o que entendemos aqui por sistemas agrários. Os sistemas agrários assentam-se sobre sistemas de produção rural: conjunto de atividades e culturas que representam um momento de uma trajetória tecnológica (Nelson e Winter, 1982; Possas, Sales-Filho e Silveira, 1996) ajustada a condições espaciais próprias de uma dada região ou lugar. Os sistemas de produção rural são o fundamento técnico dos sistemas agrários.

Há dois conjuntos principais de estruturas que se fundamentam em bases rurais no Estado do Pará, caracterizadas por relações sociais próprias, indissociáveis das relações de propriedade que lhes são subjacentes, e por relações técnicas materializadas em sistemas de produção rural também particulares. São eles: as Formas de Produção Camponesas (agrícolas e extrativas), baseadas no trabalho familiar, e as Formas de Produção Patronais - estruturas que não se reproduziriam sem o recurso ao trabalho assalariado ${ }^{1}$.

Tais formas de produção reproduzem-se condicionadas por parâmetros definidos no quadro da concorrência que se trava entre elas. O mercado é um dos "lugares" onde ocorrem tais embates. Há processos, contudo, cujo andamento se faz em outros ambientes institucionais, tanto os próprios da sociedade civil, quanto aqueles da sociedade política (Estado). Os esforços de reprodução das estruturas, que não raro exigem sua ampliação, criam os campos de força (no sentido de Bourdieu, 1983 e 1987) definidos pelo empenho de cada qual em garantir os pressupostos de recursos naturais, de

1. Para uma discussão teórica e histórica dessas estruturas, ver Costa (2000). 
trabalho, de bens tecnológicos, e de saber técnico - seus capitais específicos. O fato de esses embates fazerem-se diferenciadamente - às vezes mais em torno da terra, às vezes mais em torno do trabalho, às vezes mais em torno do mercado de produtos, às vezes mais em torno das políticas públicas - caracteriza os sistemas agrários, que são, assim, momentos particulares de uma estruturação dinâmica mais ampla, no tempo e no espaço.

As possibilidades das recomendações institucionais para o desenvolvimento seguro dependem das condições de funcionamento desses campos de força: das condições de reprodução dos sistemas agrários. Essas condições constituem as bases dos processos decisórios por meio dos quais novas técnicas e procedimentos serão absorvidos ou rejeitados. Ademais, essas técnicas e procedimentos serão tanto mais absorvidos, quanto maior o grau de convergência que tenham as oportunidades por eles oferecidas com as diversas intervenções do Estado, em que a compatibilidade de objetivos, possível pela prevalência de um projeto de desenvolvimento, é essencial. No conjunto, o papel das políticas públicas em uma sociedade baseada em mercado é o de atuar no processo decisório dos agentes particulares, reforçando ou inibindo suas inclinações no sentido de atender a objetivos definidos no processo de construção de um projeto de desenvolvimento, de algum modo percebido como orientador de um futuro melhor para todos.

O objetivo último deste trabalho é trazer elementos para discutir a esperança de sustentabilidade dos sistemas agrários presentes na Amazônia, enfocando de modo privilegiado seus sistemas de produção rural. Para tanto, far-se-á, em um primeiro momento, uma caracterização desses sistemas e seus fundamentos produtivos e reprodutivos na mesorregião Nordeste Paraense, do Estado do Pará, para, em um segundo momento, construir indicadores que possam orientar os empenhos institucionais que se pretendem voltados para a sustentabilidade. Os indicadores são construídos para os sistemas de produção rural detectados estatisticamente, de modo a incorporar tanto variáveis que enunciam esperança de maior eficiência ecológica quanto variáveis que informem sobre a eficiência econômica privada e a relevância social.

O artigo constará de quatro partes, além desta introdução. Em primeiro lugar, contextualizar-se-á a produção rural no Pará e suas formas de produção fundamentais, bem como o peso da mesorregião Nordeste Paraense no total do Estado. Em segundo, apresentar-se-ão os sistemas de culturas e atividades que fazem a base produtiva da mesorregião em questão associadas às relações de produção nela presentes. Num terceiro momento, discutir-se-á a eficiência econômica desses sistemas para, ao final, estabelecer associações entre economicidade, sustentabilidade e relevância social com o propósito de encaminhar indicadores para a orientação das políticas públicas. 


\section{Sobre as relações de produção: pondo em relevo as formas de produção e seus agentes}

Os dados aqui utilizados provêm do Censo Agropecuário 1995-96, Estado do Pará, das bases em CD-ROM postas à disposição pelo IBGE. É importante mencionar que esse meio de publicação dos dados censitários tem permitido sua utilização bem mais ampla e flexível. Não apenas pela velocidade de acesso, mas sobretudo pelo fato de permitir que se obtenham todas as tabelas-padrão que apresentam os resultados do Censo, anteriormente só disponíveis para o conjunto das unidades federativas, para todos os demais níveis regionais de agregação - até o município. Todavia, a estrutura tabelar padrão de exposição dos dados mantém um alto grau de rigidez na relação entre as variáveis. Mesmo assim, poder-se-á trabalhar com graus tanto maiores de flexibilidade, quanto mais se manejem os dados em níveis espaciais mais elementares. Isso porque, em qualquer desses níveis, essas tabelas-padrão oferecem a possibilidade de ter todas as variáveis constantes das tabelas básicas, cruzadas com os 15 estratos de área total com os quais o Censo trabalha. E, no nível geográfico em que se esteja trabalhando, sempre se poderão considerar as médias de um estrato como referidas à unidade produtiva média respectiva e, assim tratado, o estrato ganha a condição de um "caso" em um novo banco de dados passível de retabulação e processamento, tendo a freqüência do estrato como um de seus campos. Trabalhamos, aqui, com uma desagregação em nível de microrregião, para todo o Estado do Pará. Desse modo, manejamos um banco de dados de 330 casos (22 microrregiões vezes 15 estratos de área).

Essa metodologia aumentou enormemente as possibilidades de utilização das informações, permitindo não só a edição de variáveis existentes e a criação de novas variáveis e indicadores de maneira ágil e com abrangência total, como também o teste de conceituações empíricas em nível bem mais elementar, e, nesse sentido, estatisticamente bem mais abrangente do que foi possível até então. Assim, pudemos operar uma distinção bastante rigorosa entre as diversas formas de produção (camponesa e patronal, numa primeira classificação, e, dentro da segunda, patronais capitalistas e patronais fazendeiros) com base no tipo de força de trabalho utilizada. Desse modo, para cada um dos 330 casos do banco (estratos de área x para microrregião y), a) encontrou-se o valor de uma variável de força de trabalho total estimada, resultado da soma das usuais categorias "membros não remunerados da família", "assalariados permanentes" e "assalariados temporários", com uma estimativa da força de trabalho contratada na forma de empreita e de outros contratos de prestação de serviço (tomou-se

\footnotetext{
2 Esta é a maneira de operacionalizar a noção de estabelecimento camponês como aquele orientado fundamentalmente por necessidades reprodutivas de um conjunto familiar. Ver Costa (2000).

3 Para uma discussão teórica e histórica dessas estruturas, ver Costa (2000).
} 
o total de valor pago por esses contratos, dividiu-se pelo valor médio da diária prevalecente multiplicada por 300 dias médios de trabalho por ano, o que resultou no contingente absoluto de trabalhadores-equivalentes contratados nessa modalidade de serviço); b) considerou-se "camponês" o estabelecimento médio, cuja participação relativa da força de trabalho de terceiros total estimada no total da força de trabalho não ultrapassasse $1 / 3^{2}$; c) considerou-se patronal o estabelecimento médio com força de trabalho de terceiro acima de 1/3; d) nessa última categoria, considerou-se dominantemente capitalista o "caso" que apresentasse força de trabalho assalariada acima de $90 \%$ e adotou-se como fazenda os demais ${ }^{3}$.

No Censo de 1995-96, o Estado do Pará contava 206.199 estabelecimentos rurais, dos quais 195.331 atenderam nossos critérios para enquadramento como estabelecimentos camponeses. Por seu turno, 10.868 poderiam ser considerados estabelecimentos patronais, dos quais 507 empresas capitalistas e 10.361 fazendas no sentido aqui estabelecido (para estes e os próximos resultados, ver Tabela 1).

As formas de produção camponesas participam com 66\% do Valor Bruto da Produção, 37\% da área utilizada e 89\% do pessoal ocupado na produção agropecuária e vegetal do Estado do Pará. As empresas representam 10,8\% e as fazendas, 23,4\% do Valor Bruto da Produção, empregando $2,2 \%$ e $8,7 \%$, respectivamente, dos trabalhadores totais aplicados. A área apropriada por essas estruturas corresponde, por seu turno, a 28,2\% e 34,9\% do total apropriado no Estado.

As mais importantes mesorregiões do Estado no que se refere à produção rural são o Sudeste Paraense, com 30,4\%, e o Nordeste Paraense, com 28,6\% de um Valor Bruto da Produção vegetal e animal de R\$ 1,026 bilhão em 1995. Em seguida, apresentam-se Marajó, Baixo Amazonas, Sudoeste do Pará e Metropolitana de Belém, com respectivamente 12\%, 11\%, $9 \%$ e $8,5 \%$.

As meso e microrregiões do Estado do Pará são referências geográficas a partir das quais se explicitarão os sistemas agrários. Elas têm histórias próprias de conformação agrária, tendo esse fato implicações não triviais nas configurações das relações de propriedade e nas relações sociais e técnicas de produção no mundo rural paraense.

O Nordeste Paraense, caracteriza-se por ser fortemente camponês: 81,6\% do VBP animal e vegetal adveio dos seus 65.046 estabelecimentos camponeses. As estruturas patronais participam com os demais $18,4 \%$ do VBP restante, dos quais 7,7\% de 68 empresas capitalistas e 10,61\% de 1.387 unidades produtivas aqui denominadas fazendas.

A estrutura fundiária é consolidada na mesorregião Nordeste Paraense, para todas as formas de produção. As terras próprias perfazem 94\% do total de terras apropriadas e, entre as formas de produção, esse percentual situou-se entre 88 e 98\%. A forma de acesso à terra por não-proprietários é basicamente a ocupação (5,32\%). Frise-se a irrelevância das formas baseadas em pagamento de rendas: apenas 0,19\% baseado em arrendamento e $0,29 \%$ em parcerias. 
Tabela 1 - Número de estabelecimentos, valor da produção, terras apropriadas e pessoal ocupado na produção rural do Estado do Pará, 1995-96.

\begin{tabular}{|c|c|c|c|c|c|}
\hline \multirow[t]{2}{*}{ Mesorregiões } & \multicolumn{4}{|c|}{ Estrutura relativa } & \multirow{2}{*}{$\begin{array}{c}\text { Valores } \\
\text { absolutos }\end{array}$} \\
\hline & Camponeses & $\begin{array}{l}\text { Empresas } \\
\text { Capitalistas }\end{array}$ & Fazendas & Total & \\
\hline \multicolumn{6}{|c|}{ Número de estabelecimentos (Total geral $=100 \%$ ) } \\
\hline Baixo Amazonas & $13,18 \%$ & $0,00 \%$ & $0,40 \%$ & $13,59 \%$ & $28.013,0$ \\
\hline Marajó & $12,76 \%$ & $0,00 \%$ & $0,23 \%$ & $12,99 \%$ & $26.785,0$ \\
\hline Metropolitana Belém & $3,95 \%$ & $0,00 \%$ & $0,31 \%$ & $4,26 \%$ & $8.791,0$ \\
\hline Nordeste Paraense & $38,98 \%$ & $0,04 \%$ & $0,88 \%$ & $39,90 \%$ & $82.269,0$ \\
\hline Sudeste Paraense & $16,77 \%$ & $0,19 \%$ & $2,29 \%$ & $19,26 \%$ & $39.706,0$ \\
\hline Sudoeste Paraense & $9,08 \%$ & $0,01 \%$ & $0,92 \%$ & $10,01 \%$ & $20.635,0$ \\
\hline Total & $94,73 \%$ & $0,25 \%$ & $5,02 \%$ & $100,00 \%$ & \\
\hline Valores absolutos & $195.331,0$ & 507,0 & $10.361,0$ & & $206.199,0$ \\
\hline \multicolumn{6}{|c|}{ Valor da produção (Total geral = 100\%) } \\
\hline Baixo Amazonas & $9,05 \%$ & $0,83 \%$ & $1,28 \%$ & $11,16 \%$ & $114.518,5$ \\
\hline Marajó & $10,87 \%$ & $0,06 \%$ & $1,29 \%$ & $12,22 \%$ & $125.362,2$ \\
\hline Metropolitana Belém & $4,16 \%$ & $0,52 \%$ & $3,78 \%$ & $8,46 \%$ & $86.779,8$ \\
\hline Nordeste Paraense & $22,72 \%$ & $2,37 \%$ & $3,55 \%$ & $28,63 \%$ & $293.805,8$ \\
\hline Sudeste Paraense & $12,74 \%$ & $6,87 \%$ & $10,81 \%$ & $30,41 \%$ & $312.027,8$ \\
\hline Sudoeste Paraense & $6,26 \%$ & $0,19 \%$ & $2,67 \%$ & $9,13 \%$ & $93.645,5$ \\
\hline Total & $5,79 \%$ & $10,83 \%$ & $23,38 \%$ & $100,00 \%$ & \\
\hline Valores absolutos & $675.116,9$ & $111.153,7$ & $239.869,1$ & & $1.026 .139,6$ \\
\hline \multicolumn{6}{|c|}{ Terras apropriadas (Total geral = $100 \%$ ) } \\
\hline Baixo Amazonas & $5,48 \%$ & $6,04 \%$ & $2,34 \%$ & $13,86 \%$ & $3.120 .386,7$ \\
\hline Marajó & $5,72 \%$ & $0,55 \%$ & $5,68 \%$ & $11,94 \%$ & $2.689 .925,6$ \\
\hline Metropolitana Belém & $0,43 \%$ & $0,11 \%$ & $0,44 \%$ & $0,98 \%$ & $221.521,6$ \\
\hline Nordeste Paraense & $7,35 \%$ & $2,71 \%$ & $4,22 \%$ & $14,29 \%$ & $3.217 .394,4$ \\
\hline Sudeste Paraense & $11,11 \%$ & $17,19 \%$ & $16,85 \%$ & $45,16 \%$ & $10.169 .298,5$ \\
\hline Sudoeste Paraense & $6,82 \%$ & $1,61 \%$ & $5,35 \%$ & $13,77 \%$ & $3.101 .702,5$ \\
\hline Total & $36,92 \%$ & $28,21 \%$ & $34,87 \%$ & $100,00 \%$ & \\
\hline Valores absolutos & $8.313 .840,3$ & $6.353 .746,2$ & $7.852 .642,8$ & & $22.520 .229,3$ \\
\hline \multicolumn{6}{|c|}{ Trabalhadores empregados (Total geral $=100 \%$ ) } \\
\hline Baixo Amazonas & $13,06 \%$ & $0,20 \%$ & $0,57 \%$ & $13,83 \%$ & 125.396 \\
\hline Marajó & $11,85 \%$ & $0,01 \%$ & $0,39 \%$ & $12,25 \%$ & 111.117 \\
\hline Metropolitana Belém & $3,18 \%$ & $0,07 \%$ & $0,46 \%$ & $3,71 \%$ & 33.609 \\
\hline Nordeste Paraense & $34,84 \%$ & $0,39 \%$ & $1,50 \%$ & $36,73 \%$ & 333.057 \\
\hline Sudeste Paraense & $16,79 \%$ & $1,50 \%$ & $4,39 \%$ & $22,69 \%$ & 205.744 \\
\hline Sudoeste Paraense & $9,38 \%$ & $0,05 \%$ & $1,37 \%$ & $10,80 \%$ & 97.936 \\
\hline Total & $89,09 \%$ & $2,23 \%$ & $8,68 \%$ & $100,00 \%$ & \\
\hline Valores absolutos & 807.965 & 20.199 & 78.697 & & 906.861 \\
\hline
\end{tabular}

Fonte: IBGE. Censo Agropecuário do Estado do Pará. 1995-96. CD-ROM. Tabulações especiais do autor. 


\section{Sobre as relações técnicas}

\section{Os sistemas de produção agropecuários}

O que aqui chamamos de sistema de produção agropecuário foi eleito a partir da composição do VBP provindo dos oito principais grupos de culturas e atividades apresentados no Censo: as culturas temporárias e permanentes, a pecuária de grande, médio e pequeno porte, a silvicultura, a horticultura e o extrativismo.

Para "isolar" estatisticamente os sistemas, consideramos a posição relativa de cada caso em relação à média estadual de cada categoria (camponês ou patronal) para cada grupo de atividades ou culturas. Por exemplo, se em um caso já classificado como camponês, a participação relativa das culturas temporárias é superior ou igual à média dessa participação entre todos os classificados como camponeses no Estado, atribuiu-se ao seu sistema de produção rural uma característica Tmp+, isto é, a de um sistema com presença das temporárias acima da média. Para um caso em que as temporárias situam-se abaixo da média, então assinalamos Tmp-, e assim por diante.

Aplicado esse algoritmo ao nosso já esclarecido banco de dados, explicitaram-se 21 sistemas de produção rural na mesorregião: nove relativos à produção camponesa e doze, às formas patronais de produção. Destes, nove dizem respeito às fazendas e três, às empresas baseadas em assalariamento. Na Tabela 2, esses sistemas estão ordenados pela importância econômica respectiva na mesorregião, indicada, tal importância, pelo Valor Bruto da Produção correspondente. O ranking está expresso na identificação alfanumérica do sistema e precede a sua designação. Por exemplo, o sistema C1(Prm-Tmp+PecGr-PecPq-) significa sistema camponês (C), o primeiro (1) no ranking de todos os sistemas de produção na mesorregião; a importância econômica das culturas permanentes está abaixo da média estadual (Prm), as temporárias, acima (Tmp + ), a pecuária de grande porte e de pequeno porte está abaixo da média estadual (PecGr- e PecPq-).

Destaque-se o seguinte:

a) Os cinco mais importantes sistemas de produção rural da mesorregião, responsáveis por aproximadamente $73 \%$ do seu VBP, são camponeses. O primeiro deles, praticado por 38.346 estabelecimentos - dos quais 53\% na microrregião Guamá, 25\% na microrregião Tomé-Açu, 13\% na Bragantina e 9\% no Salgado (sobre a distribuição espacial, ver Tabela 3) -, representa 34\% do VBP mesorregional (ver Tabela 4, primeira coluna) e baseia-se nas culturas temporárias (shifting cultivation) seguidas das culturas permanentes (ver Tabela 2); o segundo mais importante sistema, base de 13.133 estabelecimentos - dominantemente na Bragantina (90\%), mas também na Salgado (9\%) -, com 13\% do VBP, distingue-se do primeiro pela relevância da pecuária de pequeno porte; o terceiro, praticado por 14.850 estabelecimentos - 87\% na MRH Cametá e 13\% na Tomé- 
Açu -, produz 12,7\% do VBP e caracteriza-se pela proeminência do extrativismo, que é secundado pelas temporárias e permanentes; a proeminência destas últimas é o que distingue o quarto sistema, praticado por 7.639 unidades produtivas em Cametá (58\%), ToméAçu (34\%) e Salgado (8\%), que explicam 8,6\% do VBP da mesorregião. Não obstante a variedade de composições entre os sistemas, a distribuição final das atividades e produtos entre os camponeses, na mesorregião, é de 47\% das culturas temporárias, 20\% de extrativismo, 19\% de culturas permanentes, 6\% para pecuária de grande porte e $6 \%$ de pecuária de pequeno porte. A pecuária de médio porte e os hortigranjeiros têm participações em torno de 1\%, e a silvicultura, participação próxima de zero.

b) As empresas apresentaram três sistemas de produção: o mais importante, um sistema praticado por 8 empresas de produção de aves (97\%), todas localizadas na Bragantina, cuja produção representa 5,18\% do VBP da mesorregião Nordeste Paraense; um outro, localizado em Tomé-Açu, $11^{\circ}$ no ranking do VBP, é fortemente baseado em culturas permanentes (85\%); o outro, $13^{\circ}$ em importância econômica, baseia-se em pecuária bovina (88\%), localiza-se quase totalmente na MRH Guamá. As empresas em conjunto baseiam-se 23\% em pecuária bovina, 47\% em pecuária de pequeno porte e $24 \%$ em culturas permanentes.

c) As fazendas apresentam um variado repertório de sistemas de produção. Os mais importantes deles, sétimo e nono no ranking do VBP mesorregional, são praticados, respectivamente, por 884 e 507 das 1.813 fazendas da messoregião e baseiam-se em pecuária e culturas, tanto temporárias, como permanentes, distinguindo-se um do outro pela importância da pecuária de grande porte, no caso do primeiro, e de pequeno porte, no caso do segundo. O seguinte, o $12^{\circ}$ no ranking mesorregional, conjuga pecuária de grande corte com extrativismo, e o seguinte, $14^{\circ}$ no ranking mesorregional, localizado no Guamá e em Tomé-Açu, baseia-se em extrativismo com culturas permanentes. Os demais são sistemas praticados por pequeno número de fazendas, destacando-se o elevado nível de especialização. Tomadas em conjunto, as fazendas na mesorregião dependem $42 \%$ da pecuária de grande porte, 24\% de culturas permanentes, $15 \%$ de extrativismo, 11\% de culturas temporárias e 9\% de pecuária de pequeno porte. 
Tabela 2 - Composição relativa dos grupos de culturas e atividades no VBP dos sistemas de produção rural na mesorregião Nordeste Paraense, 1995-1996

\begin{tabular}{|c|c|c|c|c|c|c|c|c|}
\hline \multirow{3}{*}{$\begin{array}{c}\text { Sistemas de Produção } \\
\text { Rural }\end{array}$} & \multicolumn{8}{|c|}{ Grupo de culturas ou atividades } \\
\hline & \multicolumn{3}{|c|}{ Pecuária - Porte } & \multirow{2}{*}{\begin{tabular}{|c|} 
Extrati- \\
vismo
\end{tabular}} & \multirow{2}{*}{$\begin{array}{c}\text { Horti- } \\
\text { granjeiro }\end{array}$} & \multirow{2}{*}{$\begin{array}{r}\text { Culturas } \\
\text { Perma- } \\
\text { nentes }\end{array}$} & \multirow{2}{*}{$\begin{array}{c}\text { Silvicul } \\
\text { tura }\end{array}$} & \multirow{2}{*}{ 1. Tempo- } \\
\hline & Grande & Médio & Pequeno & & & & & \\
\hline \multicolumn{9}{|c|}{ Formas camponesas } \\
\hline $\mathrm{C} 1$ (Prm-Tmp+PecGr-PecPq-) & $4,64 \%$ & $0,85 \%$ & $3,51 \%$ & $11,22 \%$ & $61,80 \%$ & $17,50 \%$ & $0,04 \%$ & $60,44 \%$ \\
\hline C2(Prm-Tmp+PecGr-PecPq+) & $7,87 \%$ & $0,80 \%$ & $11,36 \%$ & $8,45 \%$ & $6 \quad 1,89 \%$ & $1 \%$ & $0,01 \%$ & $52,51 \%$ \\
\hline C3(Prm-Tmp-PecGr-PecPq-) & $0,70 \%$ & $2,58 \%$ & $2,80 \%$ & $58,95 \%$ & $0,77 \%$ & $14,35 \%$ & $0,01 \%$ & $19,85 \%$ \\
\hline $\mathrm{C} 4(\mathrm{Prm}+\mathrm{Tmp}+\mathrm{PecGr}-\mathrm{PecPq}-)$ & $2,39 \%$ & $1,63 \%$ & $3,41 \%$ & $23,39 \%$ & $0,64 \%$ & $25,80 \%$ & $0,06 \%$ & $42,67 \%$ \\
\hline C5(Prm+Tmp-PecGr-PecPq-) & $7,30 \%$ & $2,29 \%$ & $4,38 \%$ & $29,31 \%$ & $0,4 \varepsilon$ & $\%$ & $0,02 \%$ & $27,63 \%$ \\
\hline $\mathrm{C} 8(\mathrm{Prm}+\mathrm{Tmp}-\mathrm{PecGr}-\mathrm{PecPq}+)$ & $11,27 \%$ & $0,70 \%$ & $24,98 \%$ & $2,92 \%$ & $1,97 \%$ & $26,21 \%$ & $0,00 \%$ & $31,96 \%$ \\
\hline $\mathrm{C} 10(\mathrm{Prm}+\mathrm{Tmp}+\mathrm{PecGr}+\mathrm{PecPq}-)$ & $27,84 \%$ & $1,09 \%$ & $2,54 \%$ & $5,38 \%$ & 0,120 & $0 \%$ & $0,00 \%$ & $35,53 \%$ \\
\hline C15(Prm-Tmp-PecGr-PecPq+) & $12,04 \%$ & $1,51 \%$ & $12,07 \%$ & $23,36 \%$ & $0,60 \%$ & $3 \%$ & $0,00 \%$ & $32,59 \%$ \\
\hline C19(Prm-Tmp-PecGr+PecPq-) & $61,69 \%$ & $0,65 \%$ & $0,67 \%$ & $5,70 \%$ & $0,83 \%$ & $8 \%$ & $3,14 \%$ & $14,74 \%$ \\
\hline Total &, $58 \%$ & $29 \%$ & $5,61 \%$ & $0 \%$ & 1,2 & $2 \%$ & $0,04 \%$ & $46,77 \%$ \\
\hline \multicolumn{9}{|c|}{ Formas patronais - Empresas } \\
\hline PE6(Prm-Tmp-PecGr-PecPq+) & $2,25 \%$ & $0,49 \%$ & $96,80 \%$ & $0,01 \%$ & $0,00 \%$ & $0,33 \%$ & $0,00 \%$ & $0,11 \%$ \\
\hline PE11(Prm+Tmp-PecGr-PecPq-) & $2,49 \%$ & $0,05 \%$ & $0,01 \%$ & $12,74 \%$ & $0,00 \%$ & $84,64 \%$ & $0,00 \%$ & $0,08 \%$ \\
\hline PE13(Prm-Tmp-PecGr+PecPq-) & $88,22 \%$ & $0,40 \%$ & $2,45 \%$ & $5,25 \%$ & $0,05 \%$ & $2,73 \%$ & $0,00 \%$ & $0,89 \%$ \\
\hline Total & $23,02 \%$ & $0,35 \%$ & $47,42 \%$ & $4,78 \%$ & 0 , & $\%$ & $0,00 \%$ & 0,2 \\
\hline \multicolumn{9}{|c|}{ Formas patronais - Fazendas } \\
\hline PF7(Prm+Tmp+PecGr-PecPq-) & $39,77 \%$ & $1,23 \%$ & $5,04 \%$ & $8,59 \%$ & 0,3 & $3 \%$ & $0,02 \%$ & $17,16 \%$ \\
\hline PFg(Prm+Tmp+PecGr-PecPq+) & $19,38 \%$ & $0,70 \%$ & $20,95 \%$ & $3,40 \%$ & $0,29 \%$ & $35,17 \%$ & $0,00 \%$ & $20,11 \%$ \\
\hline PF12(Prm-Tmp-PecGr+PecPq-) & $76,20 \%$ & $0,26 \%$ & $0,40 \%$ & $18,19 \%$ & $0,02 \%$ & $3,81 \%$ & $0,07 \%$ & $1,07 \%$ \\
\hline PF14(Prm+Tmp-PecGr-PecPq-) & $14,12 \%$ & $0,27 \%$ & $1,64 \%$ & $54,28 \%$ & 0,00 & $5 \%$ & $0,00 \%$ & $1,74 \%$ \\
\hline PF16(Prm+Tmp-PecGr+PecPq & $66,16 \%$ & $0,42 \%$ & $1,06 \%$ & $4,80 \%$ & $0,01 \%$ & $24,54 \%$ & $1,42 \%$ & $1,59 \%$ \\
\hline $\mathrm{PF} 17(\mathrm{Prm}+\mathrm{Tmp}+\mathrm{PecGr}+\mathrm{PecPq}-)$ & $69,87 \%$ & $1,18 \%$ & $1,04 \%$ & $1,54 \%$ & $0,01 \%$ & $3 \%$ & $0,00 \%$ & $8,92 \%$ \\
\hline PF18(Prm-Tmp-PecGr-PecPq-) & $48,88 \%$ & $0,06 \%$ & $0,14 \%$ & $50,72 \%$ & $0,00 \%$ & $0,06 \%$ & $0,00 \%$ & $0,14 \%$ \\
\hline PF20(Prm-Tmp-PecGr+PecPq+) & $75,25 \%$ & $0,02 \%$ & $24,59 \%$ & $0,00 \%$ & $0,00 \%$ & $0,07 \%$ & $0,00 \%$ & $0,07 \%$ \\
\hline PF21(Prm-Tmp+PecGr+PecPq-) & $87,74 \%$ & $0,00 \%$ & $0,00 \%$ & $0,15 \%$ & $0,00 \%$ & $0,00 \%$ & $0,00 \%$ & $12,11 \%$ \\
\hline Total Fazendas & $41,80 \%$ & $0,70 \%$ & $7,43 \%$ & $15,27 \%$ & $0,16 \%$ & $23,54 \%$ & $0,11 \%$ & $10,98 \%$ \\
\hline Total N & $11,51 \%$ & $1,14 \%$ & $9,29 \%$ & $18,39 \%$ & $1,13 \%$ & $20,00 \%$ & $0,04 \%$ & $38,50 \%$ \\
\hline
\end{tabular}

Fonte: IBGE. Censo Agropecuário do Estado do Pará. 1995-96. CD-ROM. Tabulações especiais do autor. Notas: PE indica que se trata de patronal empresarial ou capitalista; PF indica que se trata de patronal fazenda. 
Tabela 3 - Distribuição espacial dos estabelecimentos por microrregião e sistemas de produção rural

\begin{tabular}{|c|c|c|c|c|c|c|c|}
\hline \multirow{2}{*}{$\begin{array}{l}\text { Sistemas de } \\
\text { Produção Rural }\end{array}$} & \multicolumn{5}{|c|}{ Microrregiões } & \multicolumn{2}{|c|}{ Total } \\
\hline & Bragantina & Cametá & Guamá & Salgado & Tomé-Açu & $\%$ & Absoluto \\
\hline \multicolumn{8}{|c|}{ Formas camponesas } \\
\hline C1(Prm-Tmp+PecGr-PecPq-) & $12,59 \%$ & $0,00 \%$ & $52,93 \%$ & $9,47 \%$ & $25,02 \%$ & $100 \%$ & 38.346 \\
\hline $\mathrm{C} 2(\mathrm{Prm}-\mathrm{Tmp}+\mathrm{PecGr}-\mathrm{PecPq}+)$ & $89,75 \%$ & $0,00 \%$ & $0,00 \%$ & $8,50 \%$ & $1,75 \%$ & $100 \%$ & 13.133 \\
\hline C3(Prm-Tmp-PecGr-PecPq-) & $0,00 \%$ & $86,73 \%$ & $0,00 \%$ & $0,00 \%$ & $13,27 \%$ & $100 \%$ & 14.850 \\
\hline $\mathrm{C} 4(\mathrm{Prm}+\mathrm{Tmp}+\mathrm{PecGr}-\mathrm{PecPq}-)$ & $0,00 \%$ & $58,25 \%$ & $0,00 \%$ & $8,23 \%$ & $33,51 \%$ & $100 \%$ & 7.639 \\
\hline C5(Prm+Tmp-PecGr-PecPq-) & $0,00 \%$ & $78,60 \%$ & $0,00 \%$ & $0,03 \%$ & $21,38 \%$ & $100 \%$ & 3.869 \\
\hline $\mathrm{C} 8(\mathrm{Prm}+\mathrm{Tmp}-\mathrm{PecGr}-\mathrm{PecPq}+)$ & $72,05 \%$ & $0,00 \%$ & $0,00 \%$ & $27,95 \%$ & $0,00 \%$ & $100 \%$ & 1.170 \\
\hline $\mathrm{C} 10(\mathrm{Prm}+\mathrm{Tmp}+\mathrm{PecGr}+\mathrm{PecPq}$ & q-) $0,00 \%$ & $0,00 \%$ & $100,00 \%$ & $0,00 \%$ & $0,00 \%$ & $100 \%$ & 791 \\
\hline C15(Prm-Tmp-PecGr-PecPq+) & $0,00 \%$ & $100,00 \%$ & $0,00 \%$ & $0,00 \%$ & $0,00 \%$ & $100 \%$ & 429 \\
\hline C19(Prm-Tmp-PecGr+PecPq-) & $0,00 \%$ & $0,00 \%$ & $0,00 \%$ & $0,00 \%$ & $100,00 \%$ & $100 \%$ & 149 \\
\hline Total Camponeses & $21,72 \%$ & $25,88 \%$ & $26,23 \%$ & $7,10 \%$ & $19,07 \%$ & $100 \%$ & 80.376 \\
\hline \multicolumn{8}{|c|}{ Formas patronais - Empresas } \\
\hline PE6(Prm-Tmp-PecGr-PecPq+) & $100,00 \%$ & $0,00 \%$ & $0,00 \%$ & $0,00 \%$ & $0,00 \%$ & $100 \%$ & 8 \\
\hline PE11(Prm+Tmp-PecGr-PecPq- & -) $0,00 \%$ & $0,00 \%$ & $0,00 \%$ & $0,00 \%$ & $100,00 \%$ & $100 \%$ & 12 \\
\hline PE13(Prm-Tmp-PecGr+PecPq & q-) $0,00 \%$ & $1,67 \%$ & $98,33 \%$ & $0,00 \%$ &, $00 \%$ & $100 \%$ & 60 \\
\hline Total Empresas & $10,00 \%$ & $1,25 \%$ & $73,75 \%$ & $0,00 \%$ & $15,00 \%$ & $100 \%$ & 80 \\
\hline \multicolumn{8}{|c|}{ Formas patronais - Fazendas } \\
\hline PF7(Prm+Tmp+PecGr-PecPq-) & -) $5,32 \%$ & $16,18 \%$ & $39,71 \%$ & $12,67 \%$ & $26,13 \%$ & $100 \%$ & 884 \\
\hline PF9(Prm+Tmp+PecGr-PecPq+ & $1+) 90,53 \%$ & $0,00 \%$ & $0,00 \%$ & $9,47 \%$ & $0,00 \%$ & $100 \%$ & 507 \\
\hline PF12(Prm-Tmp-PecGr+PecPq- & -) $6,20 \%$ & $0,00 \%$ & $47,29 \%$ & $0,00 \%$ & $46,51 \%$ & $100 \%$ & 129 \\
\hline PF14(Prm+Tmp-PecGr-PecPq- & -) $0,00 \%$ & $0,00 \%$ & $0,00 \%$ & $15,29 \%$ & $84,71 \%$ & $100 \%$ & 85 \\
\hline PF16 (Prm+Tmp-PecGr+PecPa & $q-) 0,00 \%$ & $39,02 \%$ & $0,00 \%$ & $0,00 \%$ & $60,98 \%$ & $100 \%$ & 82 \\
\hline PF17(Prm+Tmp+PecGr+PecPc & $\mathrm{q}-) 0,00 \%$ & $12,38 \%$ & $87,62 \%$ & $0,00 \%$ & $0,00 \%$ & $100 \%$ & 105 \\
\hline PF18(Prm-Tmp-PecGr-PecPq-) & $\Rightarrow 0,00 \%$ & $0,00 \%$ & $0,00 \%$ & $0,00 \%$ & $100,00 \%$ & $100 \%$ & 13 \\
\hline PF20(Prm-Tmp-PecGr+PecPq & $q+) 0,00 \%$ & $0,00 \%$ & $0,00 \%$ & $100,00 \%$ & $0,00 \%$ & $100 \%$ & 6 \\
\hline PF21(Prm-Tmp+PecGr+PecPq & q-) $0,00 \%$ & $0,00 \%$ & $0,00 \%$ & $100,00 \%$ & $0,00 \%$ & $100 \%$ & 2 \\
\hline Total Fazendas & $28,35 \%$ & $10,37 \%$ & $27,80 \%$ & $9,98 \%$ & $23,50 \%$ & $100 \%$ & 1.813 \\
\hline Total Nordeste Paraense & $11,51 \%$ & $1,14 \%$ & $9,29 \%$ & $18,39 \%$ & $1,13 \%$ & $100 \%$ & $38,50 \%$ \\
\hline
\end{tabular}

Fonte: IBGE. Censo Agropecuário do Estado do Pará. 1995-96. CD-ROM. Tabulações especiais do autor. Notas: PE indica que se trata de patronal empresarial ou capitalista; PF indica que se trata de patronal fazenda.

\section{Sistemas de produção e eficiência econômica}

Na ótica dos agentes envolvidos, a eficiência econômica dos sistemas é indicada fundamentalmente pela rentabilidade líquida de cada trabalhador aplicado na produção. Formalmente,

$\mathrm{R}=\mathrm{Y} / \mathrm{T}$ ou $\mathrm{R}=(\mathrm{Y} / \mathrm{A}) .(\mathrm{A} / \mathrm{T})$ ou, ainda,

$\mathrm{R}=\mathrm{y} \cdot \mathrm{a}$

onde 
$\mathrm{Y}=$ Valor Bruto da Produção (VPB) - Custo da Produção (C)

$\mathrm{A}=$ Área total aplicada na produção

$\mathrm{T}=$ Número de trabalhadores equivalentes aplicados

$\mathrm{y}=$ Rentabilidade líquida por unidade área

$\mathrm{a}=$ Área por trabalhador-equivalente

Atente-se para o seguinte:

I. Sobre a rentabilidade dos sistemas camponeses:

a) Há uma variação importante na rentabilidade líquida do trabalho dos sistemas de produção rural, de R\$ 483 até R $\$ 1.057$ por trabalhador envolvido.

b) Com exceção do sistema C10, a eficiência econômica indicada por $\mathrm{R}$ varia inversamente com a importância respectiva no ranking medido pelo VBP.

c) Os três sistemas de maior sucesso na perspectiva da rentabilidade do trabalho são, pela ordem, o C19, o C10, o C8 e o C15.

II. Sobre a rentabilidade das formas de produção patronais empresariais:

a) Os dois mais importantes sistemas de produção empresariais apresentaram rentabilidade negativa no ano do Censo. O primeiro, baseado na produção de aves, pode ter se ressentido das novas condições impostas pelo Plano Real, deslanchado em 1994, a esse ramo produtivo. O segundo, baseado em culturas permanentes, pode espelhar a dificuldade que a racionalidade empresarial capitalista tem tido de lidar com as culturas permanentes na região (Costa, 1992, 1993 e 2000).

b) O sistema PE13, baseado em pecuária de grande porte, apresenta rentabilidade positiva.

III. Sobre a rentabilidade das formas de produção patronais fazendas:

a) Há uma grande oscilação na rentabilidade dos sistemas: há um sistema que apresenta prejuízo - o P21 - e sistemas com rentabilidade positiva por trabalhador, variando de R\$480, o P17, até R\$ 5.903, o P14.

c) Há, também, uma correlação inversa, não obstante menos intensa que a verificada para os camponeses, entre rentabilidade e importância relativa do sistema.

d) Dos dois sistemas de maior sucesso - PF14 e P20 -, um é baseado em plantio de permanentes (acima de 90\% do VBP) e o outro, em pecuária de grande porte (75\%) e pecuária de pequeno porte (25\%). Há diversos sistemas intermediários, como os de número PF12 e PF16, baseados em pecuária de grande porte, e o PF9, diversificado, apresentando, contudo, ênfase em culturas permanentes. 
Tabela 4 - Rentabilidade dos sistemas de produção rural no Nordeste Paraense

\begin{tabular}{|c|c|c|c|c|c|c|c|c|}
\hline Sistemas de produção rural & $\begin{array}{c}\text { Valor Bruto } \\
\text { da Produção } \\
\text { VBP }\end{array}$ & \begin{tabular}{|c} 
Custo da \\
Produção \\
C
\end{tabular} & $\begin{array}{c}\text { Renda } \\
\text { Liquida } \\
\text { Y=BVP-C }\end{array}$ & $\begin{array}{c}\text { Área } \\
\text { Total } \\
\text { A }\end{array}$ & $\begin{array}{c}\text { Pessoal } \\
\text { Ocupado } \\
T\end{array}$ & $Y / A=y$ & $A / T=a$ & $\begin{array}{l}\mathrm{Y} / \mathrm{T}=\mathrm{R} \\
=\mathrm{y} \cdot \mathrm{a}\end{array}$ \\
\hline C1(Prm-Tmp+PecGr-PecPq-) & 101.227 .643 & 20.014 .006 & 81.213 .637 & 463.335 & 147.608 & 175 & 3,14 & 550 \\
\hline C2(Prm-Tmp+PecGr-PecPq+) & 38.366 .516 & 11.438 .541 & 26.927 .975 & 132.589 & 55.746 & 203 & 2,38 & 483 \\
\hline C3(Prm-Tmp-PecGr-PecPq-) & 37.439 .656 & 3.750 .375 & 33.689 .281 & 74.059 & 52.385 & 455 & 1,41 & 643 \\
\hline C4(Prm+Tmp+PecGr-PecPq-) & 25.381 .542 & 5.458 .371 & 19.923 .171 & 250.387 & 31.426 & 80 & 7,97 & 634 \\
\hline C5(Prm+Tmp-PecGr-PecPq-) & 11.935 .640 & 2.473 .496 & 9.462 .144 & 86.598 & 15.404 & 109 & 5,62 & 614 \\
\hline C8(Prm+Tmp-PecGr-PecPa+) & 9.131 .480 & 3.386 .817 & 5.744 .663 & 48.210 & 6.055 & 119 & 7,96 & 949 \\
\hline $\mathrm{C} 10(\mathrm{Prm}+\mathrm{Tmp}+\mathrm{PecGr}+\mathrm{PecPq}-)$ & 6.703 .877 & 2.251 .078 & 4.452 .799 & 72.664 & 4.626 & 61 & 15,71 & 962 \\
\hline C15(Pm-Tmp-PecGr-PecPq+) & 2.333 .592 & 485.310 & 1.848 .282 & 44.845 & 2.158 & 41 & 20,79 & 857 \\
\hline C19(Prm-Tmp-PecGr+PecPq-) & 588.914 & 61.261 & 527.653 & 70 & 499 & 7.500 & 0,14 & 1.057 \\
\hline Total Camponeses & 233.108 .860 & 49.319 .255 & 183.789 .605 & 1.172 .756 & 315.908 & 157 & 3,71 & 582 \\
\hline PEG(Prm-Tmp-PecGr-PecPq+) & 11.745 .857 & 12.766 .843 & $(1.020 .986)$ & 13.421 & 357 & (76) & 37,55 & (2.856) \\
\hline PE11(Prm+Tmp-PecGr-PecPq-) & 6.687 .526 & 20.927 .749 & (14.240.223) & 238.657 & 1.635 & (60) & 145,95 & (8.709) \\
\hline PE13(Prm-Tmp-PecGr+PecPq-) & 5.848 .550 & 4.410 .143 & 1.438 .407 & 318.387 & 1.577 & 5 & 201,94 & 912 \\
\hline Total Empresas & 24.281 .933 & 38.104 .735 & $(13.822 .802)$ & 570.465 & 3.569 & (24) & 159,83 & $(3.873)$ \\
\hline PF7(Prm+Tmp+PecGr-PecPq-) & 10.480 .912 & 5.859 .630 & 4.621 .282 & 202.566 & 5.564 & 23 & 36,41 & 831 \\
\hline PFg(Prm+Tm & 9.110 .694 & 3.986 .528 & 5.124 .166 & 72.226 & 3.890 & 71 & 18,57 & 1.317 \\
\hline PF12(Prm-Tmp-PecGr+PecPq-) & 6.288 .620 & 3.184 .425 & 3.104 .195 & 233.091 & 1.465 & 13 & 159,09 & 2.119 \\
\hline PF14(Prm+Tmp-PecGr-PecPq-) & 4.628 .984 & 1.196 .151 & 3.432 .833 & 51.078 & 582 & 67 & 87,84 & 5.903 \\
\hline PF16(Prm+Tmp-PecGr+PecPq-) & 2.294 .172 & 1.340 .591 & 953.581 & 74.706 & 867 & 13 & 86,19 & 1.100 \\
\hline PF17(Prm+Tmp+PecGr+PecPq- & 2.002 .383 & 1.546 .699 & 455.684 & 65.005 & 949 & 7 & 68,50 & 480 \\
\hline PF18(Prm-Tmp-PecGr-PecPq-) & 1.094 .317 & 533.902 & 560.415 & 73.053 & 181 & 8 & 402,74 & 3.090 \\
\hline PF20(Prm-Tmp-PecGr+PecPq+) & 491.651 & 183.870 & 307.781 & 5.778 & 60 & 53 & 96,76 & 5.154 \\
\hline PF21(Prm-Tmp+PecGr+PecPq-) & 23.292 & 33.709 & (10.417) & 4.645 & 23 & (2) & 198,26 & (445) \\
\hline Total Fazendas & 36.415 .025 & 17.865 .505 & 18.549 .520 & 782.147 & 13.581 & 24 & 57,59 & 1.366 \\
\hline Total Nordeste Paraense & 293.805 .818 & 105.289 .495 & 188.516 .323 & 2.525 .368 & 333.058 & 75 & 7,58 & 566 \\
\hline
\end{tabular}

Fonte: IBGE. Censo Agropecuário do Estado do Pará. 1995-96. CD-ROM. Tabulações especiais do autor. Notas: PE indica que se trata de patronal empresarial ou capitalista; PF indica que se trata de patronal fazenda. 


\section{Rentabilidade e sustentabilidade}

A rentabilidade por trabalhador é a bússola dos agentes, sendo, portanto, R um orientador das decisões privadas que se fazem nos sistemas agrários. Nas ciências da sociedade, qualquer discussão sobre dinâmica social requer a consideração desse fato.

Há, no âmbito das ciências naturais, por seu turno, uma literatura (Svirezhev et alii, 1995; Ximenes e Van Dyne, 1999, Valdivia et alii, 1996) sugerindo, a partir da termodinâmica e de teorias de sistemas, o tratamento dos sistemas de produção rural como sistemas estritamente naturais, determinados em seu desenvolvimento pelos respectivos fluxos energéticos. Assim tratados, os agroecosistemas teriam níveis de eficiência reprodutiva e graus de estabilidade nas respectivas trajetórias de evolução estrutural correlacionados diretamente com a capacidade de aliar complexidade e densidade. E isto como resultado de leis naturais (Svirezhev, 1995: 7). Dispondo-se de uma medida de complexidade (diversidade de componentes e interações do sistema) e da intensidade energética por unidade de área, poderse-ia ter, para um agroecosistema, uma indicação de sua sustentabilidade. Melhor: um indicador da esperança de sustentabilidade física desse sistema pelo grau de sua resiliência - capacidade de absorção de impactos (Carvalho, 1994; Hobbelink, 1990; Altieri, 1989).

É exigência do ideário do desenvolvimento sustentável, contudo, que se tenha um outro plano de observação, o qual permita a leitura da maior ou menor compatibilidade entre o que move os agentes privados - a eficiência econômica, como já estabelecido - e o que garantiria um desenvolvimento amplo com qualidades antrópicas (eqüidade social) e ecológicas. Esse plano de observação deverá corresponder a uma esfera pública de atuação, dado que dele derivarão os elementos para a construção dos critérios das intervenções operacionalizadas nas políticas públicas.

Com o intuito de incorporar essas dimensões, sugerimos que a esperança de sustentabilidade seja observada por uma relação entre um indicador de confinidade (intensidade) representado pela rentabilidade (monetária) por unidade de área utilizada e por um indicador de complexidade derivado da diversidade e da eqüidade dos componentes do sistema em questão.

Sugiro, pois, uma esperança de sustentabilidade que seja:

$\mathrm{E}(\mathrm{s})=\mathrm{y} \cdot(1-\mathrm{g})$ ou $\mathrm{E}(\mathrm{s})=\mathrm{y} \cdot \mathrm{c}$ para

$$
g=\sqrt[2]{\sum_{i=1}^{n}\left(\frac{V B P_{i}}{V B P_{\text {Total }}}\right)^{2}}
$$

em que VBPi é o Valor Bruto da Produção do produto i e VBPTotal o Valor Bruto da Produção Total do sistema sob análise. O fator c seria, assim, a redundância do Índice Gini-Hirschman, g, (que é tanto maior, quanto maior 
seja o número de componentes do sistema e a eqüidade de sua distribuição), expressando o potencial de complexidade do sistema e a esperança de sustentabilidade que ele traduz como fundamento da resiliência respectiva, em relação ao impacto tanto de fatores físicos quanto econômicos. A variável y, por seu turno, é a rentabilidade por unidade de área, que expressaria o potencial de confinidade do sistema.

Sabendo-se, pela relação (1), ser

$\mathrm{y}=\mathrm{R} \cdot \mathrm{a}^{-1}$, então

$\mathrm{E}(\mathrm{s})=$ R.c. $\mathrm{a}^{-1}$ ou $\mathrm{E}(\mathrm{s})=$ R.c.e

Chegamos, assim, a uma expressão que indica o potencial de sustentabilidade de um dado sistema sendo tanto maior, quanto maiores sejam a motivação privada para geri-lo (R) e o seu potencial de complexidade (c), e quanto menor seja o volume de área requerida para o exercício do trabalho de cada trabalhador equivalente (a) - ou, visto de outro modo, quanto maior seja o inverso dessa grandeza: o número de trabalhadores que o sistema emprega por unidade de área (e).

Dado o potencial ou a esperança de sustentabilidade de um sistema enquanto artefato, resta observar a sua relevância econômico-social para estabelecer uma indicação de prioridade na ótica pública. Assim, indicar o grau de prioridade com o qual um certo sistema de produção deve ser tratado por políticas públicas que visem a sutentabilidade poderá ser possível pelo índice:

$$
\operatorname{IndP}=\operatorname{Abs}(\mathrm{E}(\mathrm{s}) \cdot \mathrm{p})
$$

Isto é: um sistema deverá ser tanto mais prioritário quanto maior o IndP, que é o valor absoluto do produto do potencial de sustentabilidade (E(s)) multiplicado pelo peso relativo que tenha o sistema de produção no sistema agrário como um todo (p). A Tabela 5 apresenta os resultados de tais proposições para o Nordeste Paraense, considerando essa região um sistema agrário. 
Tabela 5 - Indicação de prioridade no tratamento dos sistemas de produção rural do Nordeste Paraense pelas políticas públicas orientadas pelo ideário do desenvolvimento sustentável

\begin{tabular}{|c|c|c|c|c|c|c|}
\hline Sistema de Produção & $\mathrm{R}$ & $\mathrm{C}^{*}$ & $E$ & $\begin{array}{c}E(s) \\
\text { (R.c.e) }\end{array}$ & $\begin{array}{c}\text { Peso } \\
\text { Econô- } \\
\text { mico }\end{array}$ & $\begin{array}{l}\text { IndP } \\
(E(s) \cdot p)\end{array}$ \\
\hline \multicolumn{7}{|c|}{ Formas de produção Camponesas } \\
\hline C1(Prm-Tmp+PecGr-PecPq-) & 550 & 0,71 & 0,319 & 124,50 & 0,3445 & 42,90 \\
\hline C2(Prm-Tmp+PecGr-PecPq+) & 483 & 0,81 & 0,420 & 165,03 & 0,1306 & 21,55 \\
\hline C3(Prm-Tmp-PecGr-PecPq-) & 643 & 0,75 & 0,707 & 342,06 & 0,1274 & 43,59 \\
\hline C4(Prm+Tmp+PecGr-PecPq-) & 634 & 0,82 & 0,126 & 64,92 & 0,0864 & 5,61 \\
\hline C5(Prm+Tmp-PecGr-PecPq-) & 614 & 0,86 & 0,178 & 93,63 & 0,0406 & 3,80 \\
\hline $\mathrm{C} 8(\mathrm{Prm}+\mathrm{Tmp}-\mathrm{PecGr}-\mathrm{PecPq}+)$ & 949 & 0,88 & 0,126 & 104,97 & 0,0311 & 3,26 \\
\hline C10(Prm+Tmp+PecGr+PecPq-) & 962 & 0,87 & 0,064 & 53,23 & 0,0228 & 1,21 \\
\hline C15(Prm-Tmp-PecGr-PecPq+) & 857 & 0,86 & 0,048 & 35,49 & 0,0079 & 0,28 \\
\hline C19(Prm-Tmp-PecGr+PecPq-) & 1.057 & 0,57 & 7,096 & $4.280,78$ & 0,0020 & 8,58 \\
\hline Total & 582 & 0,81 & 0,269 & 126,47 & 0,7934 & 100,34 \\
\hline \multicolumn{7}{|c|}{ Formas patronais - Empresas } \\
\hline PE6(Prm-Tmp-PecGr-PecPq+) & $(2.856)$ & 0,06 & 0,027 & $(4,77)$ & 0,0400 & 0,19 \\
\hline PE11(Prm+Tmp-PecGr-PecPq-) & (8.709) & 0,47 & 0,007 & $(28,03)$ & 0,0228 & 0,64 \\
\hline PE13(Prm-Tmp-PecGr+PecPq-) & 912 & 0,74 & 0,005 & 3,36 & 0,0199 & 0,07 \\
\hline Total & (3.873) & 0,67 & 0,006 & $(16,24)$ & 0,0826 & 1,34 \\
\hline \multicolumn{7}{|c|}{ Formas patronais - Fazendas } \\
\hline PF7(Prm+Tmp+PecGr-PecPq-) & 831 & 0,92 & 0,027 & 20,94 & 0,0357 & 0,75 \\
\hline PF9(Prm+Tmp+PecGr-PecPq+) & 1.317 & 0,92 & 0,054 & 64,96 & 0,0310 & 2,01 \\
\hline PF12(Prm-Tmp-PecGr+PecPq-) & 2.119 & 0,77 & 0,006 & 10,29 & 0,0214 & 0,22 \\
\hline PF14(Prm+Tmp-PecGr-PecPq-) & 5.903 & 0,67 & 0,011 & 44,77 & 0,0158 & 0,71 \\
\hline PF16(Prm+Tmp-PecGr+PecPq-) & 1.100 & 0,81 & 0,012 & 10,36 & 0,0078 & 0,08 \\
\hline $\mathrm{PF} 17(\mathrm{Prm}+\mathrm{Tmp}+\mathrm{PecGr}+\mathrm{PecPq}-)$ & 480 & 0,86 & 0,015 & 6,00 & 0,0068 & 0,04 \\
\hline PF18(Prm-Tmp-PecGr-PecPq-) & 3.090 & 0,65 & 0,002 & 5,01 & 0,0037 & 0,02 \\
\hline PF20(Prm-Tmp-PecGr+PecPq+) & 5.154 & 0,67 & 0,010 & 35,84 & 0,0017 & 0,06 \\
\hline PF21(Prm-Tmp+PecGr+PecPq-) & (445) & 0,32 & 0,005 & $(0,72)$ & 0,0001 & $(0,00)$ \\
\hline Total & 1.366 & 0,91 & 0,017 & 21,60 & 0,1239 & 2,68 \\
\hline
\end{tabular}

Fonte: Tabelas 3 e 4 . Notas: *Este índice foi calculado considerando todos os produtos gerados pelos sistemas produtivos. Por questões de espaço, não anexamos aqui a base de cálculo. 


\section{Conclusões e recomendações}

As instituições que fazem as políticas públicas orientadas para a sustentabilidade devem considerar os sistemas produtivos em desenvolvimento, avaliando-os tanto pelo seu potencial de sustentabilidade, quanto pelo seu peso na realidade econômico-social em questão - no seu sistema agrário. Por seu turno, o potencial de sustentabilidade deve incorporar tanto a perspectiva privada de economicidade quanto os atributos sistêmicos que informam quanto à sua resiliência - diversidade, eqüidade e densidade.

Os indicadores aqui apresentados podem orientar essa avaliação. Para o Nordeste Paraense, esses indicadores apontam a premência de tratamento dos sistemas camponeses de produção: os sete maiores indicadores de prioridade recaem sobre estes sistemas: um, o de maior índice, realça o extrativismo na sua base produtiva, dois enfatizam a shifting cultivation, três tendem a se fundamentar em culturas permanentes e um, em pecuária intensiva. Por esses critérios, as fazendas apresentam um sistema interessante: o PF9, fundado numa agricultura que se compõe de culturas permanentes e temporárias, além da criação de aves. Os demais sistemas careceriam de justificação para seu tratamento. 


\section{Referências bibliográficas}

ALTIERI, M. A. (1989). Agroecologia. Rio de Janeiro: AS-PTA/FASE.

BOURDIEU, P. (1983). Questões de Sociologia. Rio de Janeiro, Marco Zero.

(1987). A Economia das Trocas Simbólicas. São Paulo, Perspectiva.

CARVALHO, H. M. (1994). Padrões de sustentabilidade: uma medida para o desenvolvimento sustentável. . In: D'INCAO, M. A.; SILVEIRA, I. M. da . A Amazônia e a crise de modernização. Belém: MPEG. p.361-380.

COSTA, F. A. (1998). Industrialism, peasant rationality and sustainable development in the Amazon. In: Liberei et alii - Proceedings of the Third SHIFT-Workshop Manaus, March 15-19. P. 219-238. . (1991).. Belém, SEPEQ/NAEA.

. (1993). Grande Capital e Agricultura na Amazônia: a experiência Ford no Tapajós.

Belém, Editora da UFPa. Ecologismo e Questão Agrária na Amazônia

. (2000). Formação agropecuária da Amazônia: os desafios do desenvolvimento sustentável. Belém: NAEA.

Bauern, märkte und kapitalakkumulation. Saarbrücken - Fort Lauderdale: Verlag Breitenbach Publishers.

GIDDENS, A. (1989). A Constituição da Sociedade. São Paulo, Martins Fontes.

HOBBELINK, H. (1990). Biotecnologia: muito além da revolução verde. Porto Alegre: s.e.

IB GE (1998). Censo Agropecuário do Estado do Pará. Rio de Janeiro: IBGE. Texto e base de dados em CD-ROOM.

IBGE (1991). Censo Agropecuário do Estado do Pará. Rio de Janeiro: IBGE.

IPEA (1999). Economia brasileira em perspectiva - 1998. Rio de Janeiro: IPEA.

NELSON, R. N., WINTER, S. G. (1982). An Evolutionary Theory of Economic Change. Cambridge, Massachussetts and London, Havard University Press.

PONTE, M. X., VON DYNE, D. L. (1999). Bioenergy Industry Analysis Based on Information Entropy. Belém: NAEA (mimeografado).

PORTER, M. (1989). A Vantagem Competitiva das Nações. Rio de Janeiro, Campus.

POSSAS, M.L., SALLES-FILHO, S. e SILVEIRA, J. M. (1996). An evolutionary approach to technological innovation in agriculture: some preliminary remarks. In: Research Policy 25(1996): 933-945.

VALDIVIA, C., DUNN, E. G., JETTE, C. (1996). Diversification as a risk management strategy in na andean agropastoral community. American Journal of Agricultural Economics 78: 1329-1334.

SVIREZHEV, Y. M., BROVKIN, V. A., DENISENKO, E. A. (1995). Agroecosistem Analysis Approach Basesd on the Flows of Artificial Energy and Information. International institute for Applied Systems Analysis - IIASA, Working Papers WP-95-27.

WILLIAMSON, O. E. (1985). The Economic Institutions of Capitalism: Firms, Markets, Relational Contracting. New York, The Free Press. 